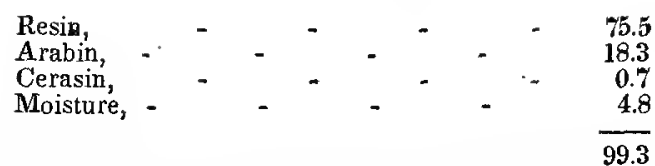

The proportion of the gum to the resin varied somewhat in each variety, but never differed more than 2 per cent. from the means given above.

The author added, that he had found the resin to be the active princjple of gamboge.

He inferred from the composition of the different kinds of gamboge, and otber circumstances detailed in his paper, that the cake gamboge of Siam is not entirely a natural production, but a manufactured article: that Ceylon gamboge, if freed from incidental fibrous matter, corresponds almost exactly with Siam gamboge: that, therefore, they are probably produced by the same plant: that Ceylon gamboge possesses precisely the same inedicinal properties: and that this variety, if more carefully collected, may, in all probability, be applied with equal advantage to every economical purpose which is at present served by the finest pipe gamboge of Siam.-Transactions of the Royal Society, 1836.

\title{
SPECIAL PATHOLOGY AND SPECIAL THERAPEUTICS.
}

22. Observations on Jaundice; more particularly on that form of the disease which accompanies the diffused infiammation of the substance of the liver. By RICHARD BRIGH', M. D.-The causes which generally give rise to jaundice will, perhaps, admit of the following classification:-

1. Congestion of blood in the liver. 2. Obstruction of bile in the biliary ducts, and more particularly in the larger ducts. 3. Chronic change in the structure of the liver. 4. Inflammatory action of the liver.

1. Congestion of blood takes place in the liver under various circumstances, of which the following are the most frequent:-Obstruction to the circulation of the chest, more particularly from valvular diseases, or dilatation of the heart; constipation, pregnancy; and certain conditions of the circulation, connected with semittent and other fevers.

2. Obstruction to the passage of the bile in the larger ducts takes place from biliary concretions; from malignant or other growths in the liver itself; or in glands about Glisson's capsule; from changes in the coats of the ducts themselves; from inflammation of the duodenum; or from induration of the pancreas.

3. The chronic changes in the structure of the liver are of various kinds; sometimes the result of simple inflammatory action of a somewhat acute character, imperfectly subdued; at other times, the result of a very slow and chronic action, affecting either the secreting portion or the cellular tissue; $\rightarrow$ at others, the result of degeneration, or of malignant action taking place extensively throughout the organ.

4. The inflammatory action of the liver, which chiefly gives rise to janndice, occurs in its substance; sometimes, if unchecked, going on to suppuration; but at other times producing a gradual change in the texture of the liver.

It is my intention, before bringing forward a few eases to illustrate the fourth class of causes to which I have referred, and wbich forms the chief object of the present communication, to state briefly some of the more prominent circumstances attending the other three classes; that, to a certain extent, we may have an opportunity of comparing, or of contrasting, their various symptoms, and the morbid changes by which they are marked.

When obstruction takes place to the circulation through the chest, but more particularly when the heart becomes gorged and over-distended with blood, we observe the countenance gradually assume a dingy aspect, in which the purple suffusion of carbonized blood is mingled with the yellow tint of a slight jaundice; tbe conjunctiva is more decidedly tinged; and if the disease continue long, the jaundice sometimes completely prevails over the purple tint: the urine becomes scanty, and high coloured, throwing down the lateritious sediments; but the dejections are not obviously deficient in bile. In this condition, the primary disease 
has occasionally been overlooked; and this error has been confirmed, when, on examination in the right hypochondrium, the liver has been found enlarged, descending three or four inches below the margin of the ribs, and decidedly tender and painful on pressure. In these cases, the obvious embarrassment in the chest, and the peculiar distress and anxiety of countenance, will generally present themselves in conjunction with the faint and dingy colour of the jaundice, as evidence of the original disease; and lead us to seek those stethoscopic signs, which will render still more obvious the nature of tbe obstructing cause.

Should death occur, it will probably have been preceded by the passage of blood, more or less freely, from the lungs or the intestines; and the examination of the body will demonstrate, that the liver has only partaken, with orher organs, in the congested state of the venous system. The liver, under these circumstances, is sometimes found in a simple state of congestion throughout all portions of its tissue, to such a degree, as to give it a general dark colour, and afford an abundant flow of blood when an incision is made; but at other times, when the congestion has been of longer duration, it presents that mottled appearance which has been correctly compared to the section of a nutmeg. In the early stages this appears to be simply a state of sanguineous congestion; but when it has continued long, some more fixed deposit seems to take place, and many of the acini assume a light-yellow colour, and a degree of firmness which is not found in the healthy organ. In connexion with this condition of the liver, the mesenteric veins will be found full of blood; the villous membrane of the intestines pretty generally of a deep-red colour, but varying in different portions; the internal surface of the stomach often suffused, to such a dcgrec, as to suggest the idea that some deleterious substance has been administered; tbe pancreas of a purple, or a leaden hue: and while thus the abdominal viscera are loaded with venots blood, the lungs are gorged, and blood is effused into them, in the form of apoplectic masses; and, in all probability, a certain quantity of serum, tinged with blood, and slightly coloured with bile, will be discovered in the cavities.

The treatment of this form of jaundice resolves itself in those means which are calculated to relieve the original disease; but much assistance is to be derived from the local abstraction of blood; by cupping from the pit of the stomach; or by the application of leeches to the anus, which more directly unloads the vessels of the abdominal circulation.

The second class of causes upon which jaundice deperids inchudes those cases in which, owing to diseased structure or vitiated secretion, some positive mechanical obstruction takes place, preventing the flow of bile from the larger ducts, and thus retaining it within the substance of the liver. In cases of this kind, we usually have a very vivid colour displayed upon the skin; which takes place either suddenly, or by slower degrees, according to the precise nature of the obstructing cause; and which continues a longer or shorter time, likewise, according to that canse; either ceasing altogether, or continuing till death takes place, at no very distant neriod; or passing gradually in to that dingy green colour which, at first sight, impresses the eye aimost like the countenance of the mulatto; and, in that sense, may not unfairly deserve the appellation of black jaundice. Amongst these mechanical causcs, the two most frequent are, undoubtedly, biliary concretions, and malignant tubera.

The presence and even the passing of biliary calculi is by no means necessarily accompanied with jaundice; for as long as the cystic duct alone is obstructed, or the hepatic or common duct only partially blocked up, the fixed yellow colour nay shew itself: although slight indications of jaundice may be present, and may recur frequently, passing away with surprising rapidity, so that almost daily changes may be observed. But, on the other hand, when the common duct is blocked up, the most brilliant jaundice often takes place, and the same in the more rare cases of the calculus being lodged in the hepatic duct. This colour will occasionally be diffused most suddenly; and when this is the case, if the obstruction be not removed, a fatal resnlt will sometimes speedily follow.

The evidences of biliary concretions, as deduced from the appearance of the jaundice, are therefore by no means determinate and certain; but the pain with which the disease is generally accompanied may be considered one, at least, of the most prominent symptoms which attend the passing of calculi, and assist in throwing light on the cause of jaundice. Tbat pain is of two kinds, - - a dull aching pain, which is constant, and an acute agonizing pain, which comes and goes 
in paroxysms. The severity of the pain is so extreme as to bring on a state of the greatest exhaustion, aud reduce the pulse below the natural standard, both as to strength and frequency, or, still more often, to render it rapid and weak, while the hands and the whole surface are bedewed with a cold perspiration. The urine becomes highly tinged with bile, and the stools of a pale drab colour; but this often varies in the course of the disease. Vomiting, hiccup, and a frequent catching inspiration often accompany this form of disease, and the symptoms are aggravated or diminished as the paroxysms of pain advance or recede. Another very frequent symptom is to be found in the occurrence of rigors at somewhat irregular intervals; but sometimes returning periodically; almost with the exactness of an intermittent. This form of jaundice is very apt to return from time to time, either from the successive passing of calculi which are seldom solitary, or from the ineffectual attempts made to get rid of a large calculus.

Should death occur in this form of jaundice, the cause of the biliary obstruction is generally detected without difficulty, by finding concretions in some of the various forms they assume, hlocking up partially, or completely, the passages by which the bile should eseape; and very frequently we have an opportunity of explaining peculiarities which bave occurred in the course of the disease, by the situation occupied by the calculus. It is by no means necessary that the calculus should be large, for we occasionally find the obstruction to have been complete, though the gall-stone has been of very moderate dimensions; and occasionally the calculus has passed, leaving the duct greatly distended, but still the constitution has not been able to rally from the effects of the disease. The liver, in this form of the disease, is found considerably loaded with bile, and the bile-ducts often distended, sometimes forming pouches in which bile is collected, shewing a longcontinued and frequently-returning tendency to obstruction.

The treatment of jaundice, under these circumstances, is to be directed to the removal of the temporary obstruction, and consists of such means as are likely to favour the passage of calculus. Opiates combined with purgatives, warm-bath, and assiduous fomentations, are amongst these means; but besides these, the use of mercurials and antimony are of great importance. The antimony, witl a view of relaxing spasms, should be combined with the purgatives, wherever vomiting is not a very prominent and distressing symptom. The use of mercury is much more doubtful, and it ought not to be carried to any great extent; for if the obstructjon be very obstinate, we run a great risk of doing mischief by over-stimulating the liver, and shall probably add to the distress under which the patient labours, both by increasing the quantity of bile secreted, when there is means of carrying it off, and likewise by increasing the irritability and diminishing the powers of the system by the action of the remedy. In these cases, then, there is no doubt that injury is not unfrequently done by the administration of mercury: but this is still more liable to be the case in tbose instances of jaundice from mechanical obstruetion, which arise from organic deposits.

The most frequent instances of organic deposit giving rise to jaundice are those in which malignant disease establishes itself in some of the complicated parts which lie in the neighbourhood of Glisson's capsule-the small lobes of the liver, the glandular structures, the pyloric end of the stomach, the substance of the duodenum, or the pancreas.

Where any organic lesion of this kind takes place, the jaundice is generally less sudden in its appearance than in other cases, though I have known apparent exceptions to this rule,--I say apparent, because, in nany cases, particularly amongst the poor, disease may have made a gradual but decided progress, without exciting attention, till the change of colour has become very observable, and then it has been supposed to have originated suddenly. It happens much more frequently that the countenance has gradually become suffused with bile, but at length the more decided jaundice has taken place, and this has gone on increasing in intensity for a time, after which the colour has lost its brilliancy, and assumed the dark dusky green hue and squalid appearance, which is one of the worst symptoms. In this form of jaundice the urine becomes loaded with bile, till it assumes a colour deeper than porter, but of a green tint; and the stools are of the lightest drab colour, approaching to white. Now, the patient becomes drowsy, ecchymosis takes place in various parts, blood escapes from different surfaces, the frame emaciates, and a state of exhaustion, succeded frequently by coma, closes the scene. 
Malignant disease may, however, exist in the liver to a very great extent, without producing any marked or decided jaundice; for as long as it developcs itself only near the surface, or in such parts as it does not make pressure on any large biliary duct, although slight effusion may result, the decided jaundice will not appcar: but, when the malignant growths occupy parts external to the liver, but are so situated as to press upon the hepatic or the common duct, this system shews itself in its greatest intensity.

There are other symptoms, more particnlarly those connceted with the evacuation from the bowels, which accompany the complete retention of the bileoccurring in cases where pressure is made on the common and perhaps the pancreatic duct--to which attention ought to be directed; though, as yet, no certain indications can be drawn from them. I refer to the evacuation of fatty matter, more or less mingled with the fæces. In several cases where the obstruction has been in the pancreas itself, a considerable quantity of that substance has been scen floating, and completely separated from the faces; of which another instance, besides those I have already published, has lately occurred to me in the hospital. But in most cases of very obstinate jaundice, accompanied by complete obstruction, an nnusual quantity of fat has been detected; and my friend, Mr. G. $\mathrm{O}$. Rees, has kindly undertaken several analyses for me, with a view of ascertaining this fact; the result of whicli may, at some future time, bc more fully stated.

The appearances presented by the liver, where organic mechanical obstruction has long existed, are generally those of the most marked accumulation of bile in all the ducts, behind the obstructing cause, whether they be the larger or the smaller, so that, in some cases, the whole liver assumes a deep olive-green colont, or occasionally a still more vivid shade of green; the large ducts within the liver are distended to the size of the finger; the hepatic, cystic, and common ducts are of still greater dimensions; and the gall-bladder, containing several ounces of bile, projects far beyond the inargin of the liver; the fundus forming a tumour, which has been perceptible to the touch, during life.

The other appearances are those which belong to the disease producing the obstruction, and to the effects of the jaundice on different organs. Amongst the former are tubera of various characters, but gencrally of hard and solid consistence, or softening towards their centre; enlarged and hardened glands; or a scirrhous state of the pancreas; and frequently a complete and permanent closing of the common ducts.

The treatment in these cases is necessarily Jittle more than palliative. As long as the symptoms have not fully convinced us of the nature of the discase, we may be induced to employ mercurials cautiously; but where the disease is confirmed, mercury can be of little or no utility, and will generally be productive of very injurious consequences: it ought, therefore, to be avoided, except in the form of an occasional purgative, and whatever can allay irritation, support the tone of the stomach, and supply gentle nourishment to the system, will afford the best merns: of prolonging life, and diminisling the suffering of the patient. What power iodine, in any of its forms, may be capable of exerting over deposits of this kind, or how far it may be able to check the progress of their formation, is at present not sufficiently ascertaincd.

The third class of causes includes various chronje changes in the struciure of the liver; and in these, many of the symptoms present a marked difference, when compared with those of the two foregoing classes. The colour of the skin seldom partakes either of the purple tinge which attends thoracic obstruction, or of the deep yellow, or the dingy green, which are so frequently observed in cases of mechanical ctosing or obliteration of the life-ducts. The change from the natural colour is usually gradual and inconstant; and the yellow tinge of the conjunctiva often precedes, for some wecks, any more decided indication. In time, however, the bronzed appearance of the forehead, or the darkened areola of the eye, bespeak the approaching change; and a jaundice, hcaring the lighter tints, from a sallow suffusion to a fainter or more decided lemon hue-still, however, liable to considerable fluctuation-establishes itsclf over the whole body.

The urine is nsually scanty, and pretty deeply coloured with bile, frequently depositing the pink sediments in abundance. The alvine evacuations seldom present that marked deficiency of bile whicl in some other cases is ubservable: on the contrary, they vary through the different shades of brown and yellow; and

No. XL.-AUGist, 1837 . 
are often remarkable, rather for the unequal manner in which the bile is mingled, than for the absence of that secretion. The action of the bowels is generally irregular; and as the disease advances, evacuations of blood frequently take place. Ascites and anasarca usually follow quickly in the train of these diseases.

The more frequent cause which exeites this form of jaundice is the excessive usc of stimulating food and drink; inducing long-continued or frequently-repeated over-action of the liver, rarely amounting to an appreciable state of inflammation. It like wise arises as a seqnel to more decided and even inflammatory attacks; and is sometimes the result of the diffused scirrhus propagating itself throngh the organ.

The appearances presented after death vary grcatly; but, as regards the liver, arc all, more or less, indicative of long-continucd morbid action. The liver is sometimes increased in its size; but very frequently quite the contrary, the organ having evidently undergone contraction in the progress of the disease: indeed I have, in some cases, most distinetly traeed its enlargement in the beginning of the attack, and its gradual diminution towards the more-confirmed stages of disorganization. Though the larger duets are pervious, and a certain quantity of imperfect bile is found in the gall-bladder. yet the whole substanee of the liver is frequently tinged by the bile retained within its smaller ducts. A general granular appearanee exists throughout the liver, as if the acini were drawn into masses, surrounded by thickened cellular membrane: and if, without being injected, a portion of liver in this state is maeerated in water for several weeks, the little yranules assume the appearance of adipocire; and may be easily washed out by a stream of water, learing a fine tissue of vessels and eellular membrane foating in the water. When the disease has gonc further, the bands of cellular membrane are seen intersecting the strueture, and forming more decided septa between the masses of acini. These are, the mure common appearanees, where, from frequent over-stimulation, gradual eliange in the structure of the liver has given jise to jaundice. Of some of the appearances produecd by malignant deposits distributed generally throughout the organ, I shall take another opportunity of speaking.

Besides the changes in the liver, a great many others, affecting the organs of the abdomen and the peritoneum, might be mentioned; but that which is most frequent is the disease and ulceration of the lining membrane of the colon, whieh very gcnerally occurs where the structure of the liver has gone into chronie degeneration; and in no case more frequently, than when the true fatty degeneration, which is seldom aecompanied by jaundice, has taken place.

The treatment in this third class of jaundice is rather by the eautious adapta. tion of food, the avoiding of stinulus, and tbe long-continued employment of such medicines as restore and keep up the moderate aetion of the system, than by any powerful remedies. Much may be done in the early stages, if the patient will submit to regimen: but when serous effusions have taken place, and other symptoms of advanced disease have shew themselves, little but temporary relief can be expected.

It will, howcver, I think: be found, that one of the most common eauses of jaundice is what 1 have assumed as a fourth cause-a state of inflammatory action more or less generally pervading the substance of the liver; and it is bighly probable, that in different eases different constituent portions of its intimate structure become more affected than the rest: bnt this is a point which requires stricter investigation than has hitherto becn bestowed upon it. I consider this inflammatory state, in many respects, different from that very chronic action of which I have lately been speaking, and which may rather be called a state of long-continucd irritation, than a state of inflammation. The causes from which it arjses are frequently very different, and, bcsides ir regularities of diet, include atmospheric exposure, the effects of external violence and injuries, the irritating effects of biliary concretions and of the retained bile, and perhaps the stimulating action of mercury. The symptoms likewise differ in many respects from those of the other forms, though not separated by sharp and abrupt lines of demarcation: and the changes produced on the substance of the liver a re decidedly different, affecting much more generally the secreting portion than the connecting cellular tissue, and probably involving the branches of the portal vein in preference to other parts. 
The progress of inflammation, in these cases, varies so greatly in its intensity and in its rapidity, as to allow of a division being made into the more and less acute forms. It frequently comes on very insidionsly, with symptoms and feelings of general constitutional derangement, depression of spirits, slow pulse, oppressed breathing, wandering abdominal pains, constipated bowels, and sometimes sickness of the stomach. In a day or two, the conjunctiva becomes tinged; and in a few days more, there is universal bright bilious suffusion of the skin. It is now found that the pulse is either accelerated, or sometimes still oppressed; and frequently, on pretty severe pressure about tbe region of the liver, some degree of tenderness is manifested; while in other cases, pressure produces little or no immediate suffering, but the pain comes on gradually a short time after the pressure has been made, and continues for hours or days. Cases of the less acute kind generally yield readily to treatment, if it be adopted early; and they form a large proportion of the cases of simple jaundice which present themselves in practice. In other cases, the infiammatory action is attended with much more severe symptoms, with considerable pyrexia, quick pulse, flushed countenance, and dry tongue, while a jaundice of the most intense colour is diffused over the whole surface. The stools are, both in the more and less acute cases, of a light colour; but less decidedly so, and subject to greater variations than when the obstruction is mechanical; and occasionally, after a few days, give little evidence of deficiency of bile. The urine is deeply tinged. When the disease assumes its more active and febrile form, those symptoms referable to the brain and nervous system, and which appear to depend upon the deleterious effects of bile circulating in the blood, are more intensely marked than in any other form of jaundice; and the tendency to hemorrhage sometimes comes on very early, and is excessive. In some cases, rigors, which assume the form of irregular intermittent paroxysms, form a prominent feature, as the disease advances; and then it often happens, though not always, that suppuration is established; and this may be going on to a great extent, while still the jaundice has rather decreased, or varied exceedingly in its intensity.

In cases of jaundice from inflammatory action, the condition of the liver after death differs according to the period at which the disease has proved fatal; but, in general, the size of the organ is not materially increased; though, on the contrary, it is not unfrequently perceptibly diminished. There is no accumulation of bile jn the minute ducts, and the yellow tinge which pervades certain portions of the structure is scarcely more than other structures of the body have obtained from the bilions impregnation with which the blood is loaded, and bears no analogy to the dark green of the liver, loaded with bile from obstruction in the large ducts. On examining the gall-bladder, it is found to contain little bile, and sometimes scarcely a trace of that fluid is to be discovered colouring the mucus accumulated by the secretion of its lining membrane.

When the disease has terminated early in its course, the whole liver seems rather soft and flaceid; the surface appears variegated, of a light-yellow, and dark-red or purple, in patches; and certain portions project above the rest, which, when cut through, sometimes prove of a softer texture, and even to be undergoing a process of change or disorganization; and portions of the same kind are intermixed throughout the whole substance of the liver; while, at other times, the yellow portions are harder than the surrounding substance.

If the disease has not proved fatal at the early period, and while the jaundice is in its brilliant and intense form, but bas gone on for some weeks, till the skin has assumed the light lemon-colour tint which often bespeaks a very general disorganization of the liver, we find the structure extensively altered, and a great many of the acini apparently altogether incapable of receiving such a quantity of blood as is necessary for the secretion of the bile, or for giving the healthy colour to the organ. They are then of a whitish-yellow colour, and rather hard and contracted than enlarged; and these altered acini are seen in groups and clusters, which, on careful examination, will generally be found to follow the course of the divisions of the portal vessels, so as to be disposed around them like a sheath, which sometimes extends to the thickness of a quarter of an inch.

There is still another condition of the liver, apparently resulting from this diffused inflammation of its substance, and which occurs when the stage of suppuration has become gradually developed; we then find the liver pervaded by a multiplicity of abscesses, all of which seem tending to discharge themselves into 
branches of the portal vein, which then assumes a most unhealthy suppurative appearance along large portions of its course.

With regard to treatment, in cases where jaundice depends upon inflammatory action, it must always be decided antiphlogistic; but it is only where it presents itself under the more violent forms, that general bleeding need be employed. In other cases, cupping from the margin of the ribs, and (as soon as the bleeding is stopped) the assidnous application of poultices over the liver, will be most important remedies. The combination of calomel, antimony, and opium, must be occasionally administered; and antimonials must be combined with the purgatives, which, in the form of pills, should be given to act regularly on the bowels, and should be aided occasionally by the sulphate of magnesia and other saline purgatives; and, in many cases, the saline purgatives, alternated occasionally with inercurials, are sufficient to cure the disease. A free and uninterrupted action from the skin is most desirable; and to promote this more effectually, the warmbath may be very advantageously employed; and the poultice, while it restrains the patient in bed, assists forcibly as a diaphoretic measure.

Under treatment of this kind, a verv large proportion of cases a re completely cured; and where the result is otherwise, it generaliy arises from some complication of diseases; most frequently from previous disorganization of the liver, or from neglect, on the part of the patient, in not applying for medical aid, or in not steadily pursuing the plan Jaid down, for it is not uncommon to find persons inclined to make jight of an attack of jaundice, if the pain or inconvenience attending is not such as to prevent them altogether from pursuing their usual occupations. Nothing, however, can be more injudicious; and it is the doty of the practitioner to impress this upon his patient; for it can never be a matter of slight importance that either obstruction or inflammatory action should exist in the intimate structure of a secreting organ, and still less in an organ so delicately complex as the liver; and there is no reason to doubt, that although, even without treatment, an attack of this kind may pass off, the liver will be some time before it has completely recovered; and thus a tendency to relapse, or to a renewal of the complaint, may occasionally be observed, shewing itself at longer or shorter intervals throughout life, and terminating a length in the destruction of all the powers of the stomach, the ulceration of the colon, miserable emaciation, or universal dropsy.-Guy's IIospital Reports, No. III.

23. Cerebral Apoplexy of New-born Infants. By Evory Knnnedr, M. D., Master of the Dublin Lying-in Hospital.- The apoplectic attack in new-born infants may be a simple primary affection, exhibiting, as we see in the following case, all the symptoms of an apoplectic seizure, as observed in the adult.

CAsE I.-Primary or Simple Apoplexy. October 14th.- The child of C. W. enjoyed perfect health up to the sixth day from birth, when it refused the breast, and suddenly fell into a state of stopor, with laboured stertorous breathing. The pulse 60; face tumid and livid; bowels, \&c. perfectly natural. One leech was immediately applied over the fontanelle, and another at the lower part of occiput; the spine was rubbed with volatile liniment, and the body immersed in a warmbath, whilst the head was kept cool. The respiration became natural, and pulse rose to 120; shortly after the leeches fell off.

15th. Much better, in every respect; its sensibilities are sufficiently acute; but still cannot suck, not grasping or holding the nipple when introduced into the mouth. To be supplied with breast milk and a small quantity of wine whey through the day. This child continued improving, and was quite well on the 16 th.

This case may be looked on in every respect as one of simple apoplexy; and it may be fairly inferred, that the train of symptoms depended upon mere congestion.

Apoplexy may be combined with inordinate or spastic action of the muscles of the face, trunk, or extremities, more or less complete or partial, a striking instance of which we have in the following case.

Case II.-Apoptexy combined with Tonic Spasm. This ehild exhibited a tumour on the occiput, the effect of a twenty-nine hours' pressure in labour. A few hours after its birth it became insensible, its respiration laboured, and the muscles of the neck and lower extremities were spastically fixed, producing complete opis- 